\title{
Letter processing and font information during reading: Beyond distinctiveness, where vision meets design
}

\author{
Thomas Sanocki • Mary C. Dyson
}

Published online: 20 October 2011

(C) Psychonomic Society, Inc. 2011

\begin{abstract}
Letter identification is a critical front end of the reading process. In general, conceptualizations of the identification process have emphasized arbitrary sets of distinctive features. However, a richer view of letter processing incorporates principles from the field of type design, including an emphasis on uniformities across letters within a font. The importance of uniformities is supported by a small body of research indicating that consistency of font increases letter identification efficiency. We review design concepts and the relevant literature, with the goal of stimulating further thinking about letter processing during reading.
\end{abstract}

Keywords Letter identification - Letter perception - Font . Font tuning · Common features · Type design · Reading

Motivated by the increasing realization that letter perception is an important but overlooked stage in the reading process (e.g., Finkbeiner \& Coltheart, 2009; Grainger, 2008; Massaro \& Schmuller, 1975; Pelli, Burns, Farell, \& Moore-Page, 2006), there has been a resurgence of interest in letter perception in relation to reading. It is now clear that letter perception provides a critical front end for reading because letters are functional units; they are independent pieces of the word code (e.g., McClelland, 1976; Oden,

T. Sanocki $(\square)$

Department of Psychology, PCD 4118,

University of South Florida,

Tampa, FL, USA 33624

e-mail: sanocki@usf.edu

\section{C. Dyson}

Department of Typography \& Graphic Communication,

University of Reading, 2 Earley Gate, Whiteknights,

Reading RG6 6AU, UK

e-mail: m.c.dyson@reading.ac.uk
1984; Pelli, Farell, \& Moore, 2003). The visual forms of letters exist within a larger structural design, a family of objects known as the type font. This idea has implications for perceptual identification that we begin to develop here.

In previous research and theory, a core concept is distinctiveness - the properties that make one letter easy to discriminate from its alternatives in the alphabet. This has led to the central concept of feature detection in the literature (e.g., Fiset et al., 2008; Gibson, 1969; Massaro \& Schmuller, 1975). Letters are defined by sets of features whose membership is determined by distinctiveness. If distinctiveness is indeed critical, increasing it through alphabet design should increase legibility. This logic has been recently advocated (e.g., Fiset et al., 2008; Gosselin \& Tjan, 2008). ${ }^{1}$ However, if letter distinctiveness is an incomplete basis for understanding letter processing during reading, calls to redesign letters are premature.

A richer view of letter processing incorporates structural relations between letters and originates in the field of type design. Type designers have long been concerned with letter form and its impact on reading. Text fonts are designed for reading continuous paragraphs of text, and the main goal in their design is to produce optimally legible letter forms. Type designers recognize the importance of distinctiveness, but they also emphasize the uniformity of letters (e.g., Carter, Day, \& Meggs, 1985; Cheng, 2005). The classical goal of

\footnotetext{
${ }^{1}$ The idea of improving legibility through research and design is not new. Modifications to increase distinctiveness have been explored by Kolers (1969), Lockhead and Crist (1980), and, more recently, Beier and Larson (2010). A modification that was actually used (mainly to increase spelling regularity, but distinctiveness was also increased) was the Initial Teaching Alphabet (Pittman \& St. John, 1969); this modification was not successful (e.g., Downing, 1967). Spencer (1968) cites proposals for new designs going back to 1881 .
} 
Fig. 1 Examples of three parameters that distinguish among fonts while relating letters within a font

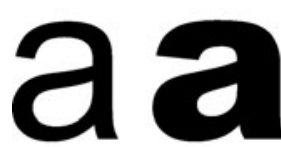

differing

weights

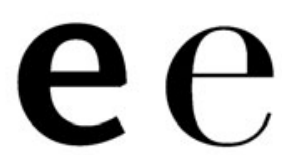

low contrast between strokes high contrast between strokes

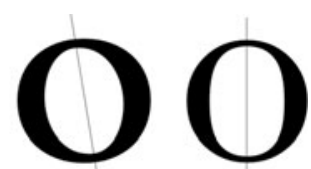

angle of stress type design is to achieve harmony and balance between individual forms. Within words, a letter should never stand out; it should cohere with neighboring letters, in order to better form a word unit and sublexical units as well. ${ }^{2}$

In type design, there is a tension between considerations of distinctiveness and uniformity that is essential to the design process. "Individual characters must be distinct, yet related, in their form and construction" (Cheng, 2005, p. 6). Uniformity is achieved through commonalities in the shape, proportions, and other stylistic attributes of letters within a font. Type designers constrain the shape of individual letters within a font so that they are related in weight, contrast, and stress, or axis, of the letter. Figure 1 illustrates these parameters. When type designers create letterforms, they develop a consistent treatment of part primitives and strokes (Adams, 1989). From a psychological perspective, the consistent and coherent appearance of fonts can be described as the application of rules, by the type designer, for "translating the prototypical structural features of every letter of the alphabet into a printed surface form" (Walker, 2008, p. 1024).

Thus, commonalities within and between letters are a design feature of high-quality text fonts. Type designers incorporate commonalities because they believe they are important for legibility, on the basis of their data. Their data are judgments refined through training, aimed at understanding the structural relations that constitute a legible font. Type design results from a design process in which design possibilities (variations in visual structure) are evaluated by the designer and other educated readers by intuitively monitoring their own reading experience.

The idea that commonalities are important in letter perception receives validation from a small body of psychological studies in which commonalities increase the efficiency of human letter identification. This research will be reviewed, and the importance of specific commonalities will be discussed. To anticipate, we conclude that the

\footnotetext{
${ }^{2}$ In general, type designers (mistakenly) assume that words are the functional units of reading. Psychological research, however, indicates that readers also form units at sublexical units such as letters, syllables, and perhaps bigrams (e.g., Grainger, 2008). Fortunately, designers work with letters and attend to how well letters form word units. We suggest that well-designed letters form functional sublexical units, while also forming good word units, which are most important for lexical access.
}

commonalities that have been supported most strongly are spatial parameters concerning the size of letter parts and their interrelations. In type design, letters of the same font use a systematic reference frame of ascender line, $x$-height, baseline, and descender line, illustrated in Fig. 2. This frame system constrains size proportions within the font ${ }^{3}$; for example, the ratio of $\mathrm{x}$-height to cap height and the length of ascenders and descenders are characteristics of a particular font (Baines \& Haslam, 2005). The proportions vary somewhat among fonts, but within a restricted range, making fonts of the same point size appear larger or smaller (Luna, 1992).

The psychological evidence that commonalities contribute to efficiency comes from advantages found in identification of letters of consistent, regular fonts, relative to mixed or irregular fonts (Gauthier, Wong, Hayward, \& Cheung, 2006; Sanocki, 1987, 1988, 1991b, 1991c). The interpretation of these results is that letter processing becomes more efficient because the perceptualprocessing system tunes itself to exploit regularities of a font (see also Sanocki, 1991b, 1992; Walker, 2008). In contrast, in mixed font conditions, although there are often the same $n$ alternative forms and letter identities, they are from two or more fonts, instead of a single font. Note that mixing fonts increases the distinctiveness of individual letters, because differences between fonts correspond with letter identities. However, spatial and other properties are not as regular in a mixed font, and the perceptual system cannot exploit them as well. As a result, letter identification efficiency is reduced relative to same-font conditions. These regularity effects imply that shared properties within a font (commonalities) are important in letter processing, in addition to distinctiveness. These commonalities are constraints that create a family of objects for identification. The ability to exploit commonalities is a hallmark of expertise with letters; nonexperts (i.e., those unfamiliar with the writing system) do not exploit regularities (Gauthier et al., 2006).

\footnotetext{
${ }^{3}$ This is not done in a precise (mathematical) way. In fact, some shapes are given different heights in order to appear equivalent in height (e.g., to match the perceived height of a curved vs. a straight line, rounded letters such as lowercase $a, c, e, o$ are usually slightly taller than $v, w, x, y$, etc.). Also designers may introduce some slight irregularities to reflect their particular design style.
} 
Fig. 2 Reference frame for letters

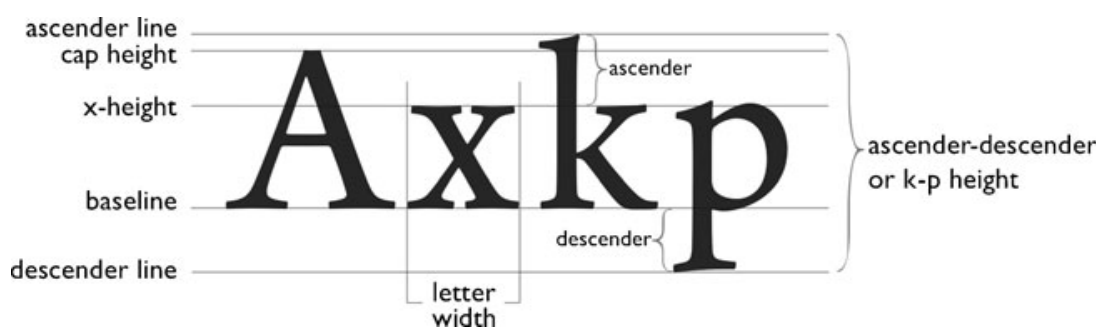

\section{Plan of this article}

We begin with preliminary issues and the idea of distinctive features. Next, we review selected recent work and contrast it with our emphasis on commonalities. In the main section, we review the small body of research on font mixing and identify outstanding issues. We conclude with several further issues.

\section{Observing and appreciating the complexity of letter processing during reading}

Letter research can benefit from discussion of the perceptual problems (Marr, 1982) that must be solved in letter processing during reading. We begin, however, by commenting on how letter stimuli should be presented to maximize experimental sensitivity to visual processing.

Because words are highly meaningful units in reading, it is natural to use them to study reading. However, higher level units in language impart strong benefits on the processing of their constituents; in particular, word unitization in the brain strongly benefits letter processing. The benefits occur because information is integrated across letters in words (e.g., Massaro \& Sanocki, 1993; McClelland \& Rumelhart, 1981), making the perceptual system more robust to limitations of letter-level processing (e.g., Oden, Rueckl \& Sanocki 1991). Sublexical units such as syllables and bigrams are also likely to cause top-down effects, as are wordlike nonwords (e.g., Grainger, 2008; McClelland \& Rumelhart, 1981). To maximize sensitivity to visual processes, these top-down effects must be obviated. One way to do so is to present unrelated letter strings as stimuli. Such strings benefit much less from higher level processing, and, critically, they embody difficulties that occur for letters in words. There is evidence from a variety of paradigms of reductions in top-down benefits as higher level units are removed. ${ }^{4}$ Of

\footnotetext{
${ }^{4}$ These include paradigms studying the identification of letters in words and nonwords (e.g., McClelland \& Rumelhart, 1981; Reicher, 1969; Wheeler, 1970), experiments on the effects on identification of visual feature information (e.g., Lanthier et al. 2009), and research on type factors such as case alternation (e.g., Mayall, Humphreys, \& Olson, 1997).
}

course, if the top-down influences on letter processing are of interest, wordlike stimuli are appropriate.

When letters are presented in strings, there are at least three classes of problems that the perceptual system must solve. Commonalities within a font may contribute to solving these problems.

Location and position uncertainty Uncertainty related to horizontal position is considerable during reading for at least two reasons. First, uncertainty is produced because the eyes move rapidly across text, landing briefly at various positions within words. Information must be registered somehow relative to eye position. Second, there is uncertainty produced by the letters themselves. Skilled readers almost always read proportionally spaced fonts, in which letter width is highly variable. As many as three narrow letters (e.g., ill) can fit within the space of one wide letter (e.g., $m$ or $w$ ). Research has documented the location uncertainty of letters when words are processed (e.g., Davis \& Bowers, 2006; Gomez, Ratcliff, \& Perea, 2008; Mozer, 1983). These complexities necessitate that the reference system for letters in words include horizontal positional information. In designing type, horizontal parameters determine the width of individual letters and the positioning of letters (including space between letters and words). Vertical position is a likely separate but also important issue. ${ }^{5}$

Crowding Crowding is caused by limitations in the visual system's ability to resolve features within particular regions, a limitation that increases with eccentricity (Pelli et al., 2007; Stuart \& Burian, 1962). Although fixated letters may not be crowded, there is increasing crowding for letters away from fixation (Pelli, Palomares, \& Majaj, 2004; Pelli et al., 2007), and crowding may be especially problematic for parafoveal processing of letter and word information (e.g., Juhasz, Pollatsek, Hyönä, Drieghe \& Rayner 2009). Thus, crowding is a further problem for letter processing during reading. Interestingly, crowding can be reduced by system-

\footnotetext{
${ }^{5}$ For example, the space between lines (referred to as interlinear or line spacing, leading, or vertical word space) affects reading speed (Chung, 2004; Paterson \& Tinker, 1940). Efficiency may be influenced by transitions between lines, because greater leading eases location of the next line (Paterson \& Tinker, 1940), and efficiency may be influenced by crowding (Chung, 2004; see the next section).
} 
atic configuration of structures (Livne \& Sagi, 2007). Crowding is also affected by whether a target blends with flankers (Saarela, Sayim, Westheimer, \& Herzog, 2009). Font design may balance these effects, providing systematicity that reduces crowding, while framing visual distinctions that aid identification. For example, ascenders and descenders are likely to be easy to distinguish because they occupy the lesscrowded upper and lower regions of letter space.

Composing a parallel cue During reading, the desired result of letter processing is lexical access, via a code that accesses the word and its meaning. In most cases, each letter has a functional role in composing the word code; skilled readers process letters in a parallel manner (e.g., Spinelli et al., 2005) and are disrupted by missing letters (e.g., White, Johnson, Liversedge \& Rayner 2008). Thus, the best word cue includes an appropriately ordered set of letter identities. This means that the relative timing with which letter information becomes active in the mind is critical; if letters within a word become active at different times, the cue is wrong and will access the wrong word(s). There is evidence of the negative effects of salient sublexical groups in research on identifying case-mixed words (Humphreys, Mayall, \& Cooper, 2003; Mayall, Humphreys and Olson 1997) and direct evidence in size-mixed letter identification (Sanocki, 1991b), discussed below. Fonts are designed to ensure that particular letters are not more salient than others; this and other design features may help ensure that letters in words become active in mind in parallel and over a similar time course.

Finally, we argue that lowercase letters are the best stimuli to study in most instances. Lowercase is the regular practice in texts meant for reading. The variation in basic letter shapes is a potent cue to identity. Thus, lowercase letters are best for studying skilled letter processing in general. All uppercase text has been found to be read slower than lowercase and is thought to be less legible (Tinker \& Paterson, 1928). ${ }^{6}$

\section{Time course of processing individual letters during reading}

We briefly summarize the time course. Theories posit a hierarchy of processing in which letter identity is quickly abstracted from visual details (e.g., the letter "a" is

\footnotetext{
${ }^{6}$ In some cases, such as low vision, uppercase may be advantageous, perhaps because of its increased size relative to lowercase (Arditi \& Cho, 2007). Also, familiar acronyms are processed more quickly in the familiar uppercase than in lowercase (Besner, Davelaar, Alcott, \& Parry, 1984; Seymour \& Jack, 1978); these results support the idea of using the most frequently encountered forms in studying normal letter processing.
}

abstracted from font and case [A, a]; e.g., Grainger, 2008; Massaro \& Schmuller 1975). That is, letter identification begins with the processing of visual information such as features, but visual details are discarded as soon as an abstract identity is extracted. Visual processing may involve global-to-local or coarse-to-fine stages, as we describe later (e.g., Fiset et al., 2009; Navon, 1977; Sanocki, 1991a, 1993, 2001). Abstract letter identities (and associated phonology) are used to identify words. The importance of abstraction is supported by the rapidity of perceiving abstract letter identity, which is independent of visual structure (Friedman, 1980).

Our main emphasis will be on the processes leading up to the activation of letter identity. The general characteristics of these processes is widely agreed upon in the research community. There is also evidence that type information can sometimes linger in word processing, becoming part of the memory code (e.g., Goldinger, Azuma, Kleider, \& Holmes, 2003); this is an interesting issue but is not treated here. In the review section, we emphasize processes leading up to identification, as opposed to decision processes that may occur after initial letter identification (cf. Neely, 1991; Sanocki, 1987, 1992).

\section{Research on letter perception}

How are letters perceived? This issue has been examined for many years, and the dominant framework is based on features - the idea that a letter is perceived by detecting independent features. The features are an arbitrary set whose combination serves to distinguish the letter, activating an abstract letter code (e.g., Fiset et al., 2009; Gibson, 1969; Massaro \& Schmuller, 1975). Accordingly, research has sought to determine what the features of letters are. Much of this research has presented individual letters, under the assumption that the features used in recognizing isolated letters are also used for letters in context. We first summarize some core ideas in this research and then go on to more recent statements.

\section{Feature detection}

The traditional method for investigating letter features has been to present letters briefly for identification and explore confusions among the letters, under the assumption that similar letters will be confused with each other (e.g., Bouma, 1971; Gervais, Harvey, \& Roberts, 1984; Gibson, 1969; Harris, 1973; Keren \& Baggen, 1981). Similarity is assumed to be determined by shared letter features, and the pattern of interletter confusions has been used to determine what the features of letters are. However, after decades of research involving confusion matrices, little agreement has 
been reached on a definitive set of features. Summarizing more than 70 published studies on letter confusability, Grainger, Rey, and Dufau (2008) described the features as "mainly consisting of lines of different orientation and curvature" (p. 381). These are local features. In contrast, Bouma (1971) proposed global features, such as vertically ascending and descending parts, slenderness, and outer parts as features that might serve as perceptual cues. Thus, a very basic question remains open, as to whether features are global or local in nature.

The value and appropriateness of using confusion matrices to identify letter features has been questioned. Pelli et al. (2006) referred to limited success in identifying features, singling out just two global attributes as important: roundness and letter width. However, common manipulations (low contrast and rapid presentation) make low spatial frequencies appear more important and, hence, are more likely to reveal global features (Fiset et al., 2008). Grainger et al. (2008) and Fiset et al. (2009) noted that letters must be degraded to create confusions, and this can influence the nature of the confusions. In fact, support for this argument was obtained by Bouma (1971), who compared two different manipulations (long reading distance and eccentric vision) and found differences in the pattern of confusions. Differences have also been attributed to the particular fonts tested; Gilmore (1985) noted that fonts vary in the spatial frequency and phase spectra of the letters due to their shape, proportions, and other stylistic attributes.

\section{Classification image technique}

In two recent articles, Fiset et al. (2009; Fiset et al., 2008) used classification image methods to discover the features used in letter identification. The method directly tests the effects on letter identification of feature samples at multiple scales. Thus, the method addresses the issue of feature scale (and spatial frequency) by examining different combinations of scaled features. On each trial in the experiments, a different combination of feature samples at multiple scales is generated, combined, and then presented as a stimulus (one stimulus centered on fixation) for subjects to identify. Then identification probabilities are analyzed with multiple regression to determine how strongly feature samples are associated with correct identification. For lowercase letters (Arial font), Fiset found that line terminations (the ends of letter parts) were by far the most important feature for human observers and much more important than the second feature, horizontals.

Fiset et al. (2009; Fiset et al. 2008) argued that terminations may be important because they represent critical identification information across most fonts-fontinvariant information. We agree with this conclusion but suggest that the perceptual importance of terminations can be understood more deeply in terms of their role within the system of distinctions within a font; terminations distinguish between different basic letter parts or their combination and, thus, between letters (e.g., Sanocki, 1987). For example, $n$ and $r$ both have a curved component (arch), but the curve of $n$ continues further into a vertical before terminating. In type design, these distinctions are systematically constrained within and across fonts and often are marked with serifs (small lines at the end of strokes) or other details.

One potential problem with single-letter studies is that absolute locations within letters are likely to be overemphasized. Over trials, the letters are superimposed over each other (presented centered in the same position); this can make letter parts that do not overlap (or overlap less) with other letters especially salient. For example, $p$ 's descender becomes a unique area of nonoverlap in the lower left of absolute letter space. In contrast, during reading, letter perception is complicated by spatial uncertainty that is resolved over time, and there is no such thing as absolute location or area of nonoverlap.

\section{Efficiency of letter processing: 1. Letter templates}

Letter processing is highly efficient in skilled reading; indeed, efficient letter processing is a requisite for comprehension (e.g., Laberge \& Samuels, 1974). Therefore, the efficiency of letter processing is an important concern. Using psychophysical methods, Pelli et al. (2006) recently developed an empirical definition of efficiency that involves an ideal observer. The measure compares the efficiency of identification by human observers with a maximum level possible for the set of stimulus alternatives, defined by an ideal observer (Tanner \& Birdsall, 1958). The ideal performance level is based on the assumption that maximal identification (optimal use of stimulus information) is achieved by comparing the stimulus representation on each trial with templates for the alternatives (in an absolute location space) and picking the alternative with maximum overlap.

Different fonts vary in the ideal level they allow, because the letters within the fonts can be more or less similar to each other. The ideal observer method factors out the overlap between letters and establishes the ideal level for each font. Human performance can then be compared with the ideal level possible with a font.

Pelli et al. (2006) established the ideal level for a number of different fonts by simulating, through a computer program, the ideal identification for single letters presented centrally but embedded in white noise. For comparison, they also calculated ideal performance for sets of alternatives consisting of simple shapes (subsets of squares in matrices - Checkers font), and sets of many words (treated as single characters). Pelli et al. (2006) then measured 
human performance with the same stimuli and presentation method and reported the ratio of human performance to ideal performance. ${ }^{7}$ Interestingly, overall efficiency was surprisingly low, ranging between 0.09 and 0.14 for typical text fonts (Bookman and Helvetica, respectively). Efficiency reached its highest level for bold fonts (Bookman Bold, 0.155) and was markedly higher for the fairly simple shapes $(2 \times 3$ Checkers, 0.308).

If efficiencies had approached 1 for some fonts, Pelli et al. (2006) would have argued that letter identification involves something like the template-matching scheme modeled by the ideal observer. Indeed, they had expected such a result. However, the low efficiencies imply, instead, that observers use an alternative and less efficient scheme. On the basis of further analyses, Pelli et al. (2006) concluded that letter identification follows an early feature detection stage involving multiple independent features. The separate feature decisions made at this stage produce the low levels of efficiency observed. The idea of an initial feature detection stage is consistent with the prior literature on feature detection as the basis of letter identification.

Pelli et al. (2006) have developed a measure that is formal, specific, and useful in certain ways. However, the Pelli et al. (2006) approach is limited as well. One general problem is that efficiency was low for high-quality, common text fonts; these results call into question the validity of applying this overall measure to reading. The Checkers font, made of randomly combined squares, had the highest efficiency.

Why might text font efficiency be so low? Simple problems include differences caused by brief stimulus presentations (in contrast to reading) and presenting individual letters centered at fixation, which may overemphasize exact spatial information. More important for this article, the concept of efficiency, as well as the ideal observer model, is based on the assumption that distinctiveness (template differences between letters in absolute space) is critical and that only distinctiveness is critical. The assumption is inconsistent with the basic assumption in type design that commonalities among letters are also important. Note that distinctiveness could be increased by creating a mixed font, where differences between fonts would increase interletter distinctiveness. For such a font, ideal observer efficiency should go up. Yet this would seriously contravene type designers' intuitions as to what is a legible font. Furthermore, evidence indicates that letter perception would be slowed down by font mixing.

A related point is that the ideal observer approach ignores another possible basis of efficiency during reading.

\footnotetext{
${ }^{7}$ In more detail, Pelli and colleagues reported a refined and advantageous version of this concept, high-noise efficiency (Pelli \& Farell, 1999).
}

In almost all texts, the font remains the same within words and throughout the text. Commonalities of type could be exploited by the perceptual system. Letters within a string share spatial parameters (such as $\mathrm{x}$-height or ascender height), and their reference frames may be resolved in parallel over time. And, because the parameters of a good font remain constant, much of the perceptual information used for one word could be applied to subsequent letter processing. Thus, letter-processing efficiency could be increased by tuning of the perceptual system.

Efficiency of letter processing: 2 Parts, relations, and font tuning

Independent features are an elegant way to decompose letters into psychologically functional subunits, but they are not the only analytic approach. An alternative approach to letter-processing efficiency that incorporates type commonalities (Sanocki, 1987) has been developed using structural network concepts, from the literatures on modeling visual knowledge about objects and scenes (schema theories; see, e.g., Oden, 1979; Palmer, 1975; Pinker, 1984; Sanocki, 1999), including letters (Knuth, 1982). In the network approach, object structure is modeled in terms of entities and relations between the entities that are made explicit within a network representation (e.g., Oden, 1979). In type, the major component entities of letters may be letter parts (Sanocki, 1987). Relations and commonalities among components are made explicit in the network, including spatial size parameters, part shape, and details of lines and terminations (Knuth, 1982; Sanocki, 1987). ${ }^{8}$

The design of some subtle details of fonts can be seen for four well-designed text fonts in Fig. 3. Consider, for example, the terminal elements on $a, c, f$, and $r$; these are consistent within font but vary in style between fonts. Similar differences involve the termination of the ascenders $(d, h, n)$ and the extent of the descenders on $j$ and $y$. Serifs, of course, differ systematically, as do the weight and height of dots $(i, j)$. The relations between these details can be represented in a network. More important for reading, fontspecific details could be a basis for tuning within and across strings. The idea of tuning leads to novel predictions about the positive effects of uniformity on letter-processing efficiency. These predictions have been examined with

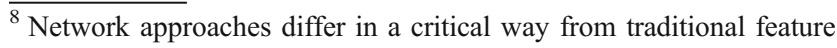
models. Traditional models assume that features are both the unit of extraction from the stimulus and a component of the letter representation. Network models emphasize the information in the representation; the units of extraction are a separate issue. (Although not a necessary assumption, the units of extraction could consist of information about edge pieces and relations between line pairs that are interpreted within the network, for example.)
} 


\section{adhefcrnijy adhefcrnijy adhefcrnijy adhefcrnijy}

Fig. 3 Examples from four popular text fonts (Bookman Old Style, Optima, Times, and Helvetica)

differences between fonts that are greater than the details illustrated here.

The efficiency of a consistent font

The possibility of tuning for font-specific details led Sanocki $(1987,1988)$ to create contrasting fonts and vary the consistency of the font within strings and between strings. Sanocki $(1987,1988)$ measured the effects of font consistency on general measures of letter identification efficiency. The importance of font consistency was implicated by prior reading research indicating more efficient perception when typographic variables such as size, case, and type style are consistent (e.g., Corcoran \& Rouse, 1970; Rudnicky \& Kolers, 1984; Tinker, 1963). However, the focus on letter identification by Sanocki was new.

Sanocki (1987) developed fonts that were similar in overall letter size (top of ascender to bottom of descender) but differed on several properties, including the extent and details of terminations, the basic shape of parts (Experiments 1-3), and line thickness and spatial dimensions ( $\mathrm{x}-$ height, ascender/descender height; Experiment 2). Strings of letters were presented, and the font was either consistent within strings or mixed within strings. Sanocki (1987) used a letter-nonletter task; the letter strings sometimes contained a nonletter (a letter with a part added or deleted), and observers pressed a key to indicate their judgment. Responses were considerably faster for same-font strings over mixed strings (over $100 \mathrm{~ms}$ in most cases). Analyses based on additive-factors logic (Sternberg, 1969) implied that the same-font advantage arose from the speed of activating letter codes, rather than response or decision processes. The large font-mixing effect was additive with other large effects, presumably because it involved visual processes preceding checking or decision processes. The other effects on response time were produced by the factors string length and response (letter-nonletter); string length caused linear increases, while response caused a 2:1 slope pattern (Sanocki, 1987, Experiment 2). The conclusion that font mixing affected the efficiency of letter activation was supported more directly in the next set of studies.

Sanocki (1988) used fonts similar in overall size but differing on several properties; letters from these fonts are shown in Fig. 4. The letters were presented briefly in strings, followed by a pattern mask and then a measure of accuracy of letter identification-accuracy of a forced choice between two same-font letter alternatives at each string position. This is a fairly direct measure of the efficiency of the letter identification processing. Font was the same within strings or mixed in either of two ways. Font was mixed within strings in Experiment 1, whereas in Experiment 2, it was mixed either within strings (for half of the observers) or between strings (alternating between the two fonts on every trial). The letter strings were presented for varying amounts of time to produce accuracy functions. The functions indicated that identification was more accurate in the same-font conditions, especially since accuracy rose with longer presentation time. The advantage for same-font conditions held for mixing within and between strings, although the mixing decrement was (nonsignificantly) larger when font was mixed within strings. The advantage for same-font conditions was also generally constant across letter position. The results indicate that font consistency influences the speed with which letter codes become activated (Sanocki, 1988) and converge with the conclusions from the reaction time task (Sanocki, 1987). Font-mixing decrements have been replicated and extended in further research by Sanocki (1991b, 1991c, 1992) with both tasks. Also, these studies have produced same-font advantages while equating number of letter instances (Sanocki, 1987, 1988) and number of letter parts (Sanocki, 1987, Experiment 2) between conditions.

The fonts in these studies were created by Sanocki, within dot matrices that were reasonable for the computer technology at that time $(20 \times 8$ pixels, total height $\times$ width $)$. The fonts are not as high in visual quality as typical text fonts in current use. Do the negative effects of font mixing generalize to higher quality fonts? More recent work with improved letter displays begins to address this issue (Gauthier et al., 2006; Walker, 2008). Perhaps most compelling is Gauthier et al.'s Experiment 3, which

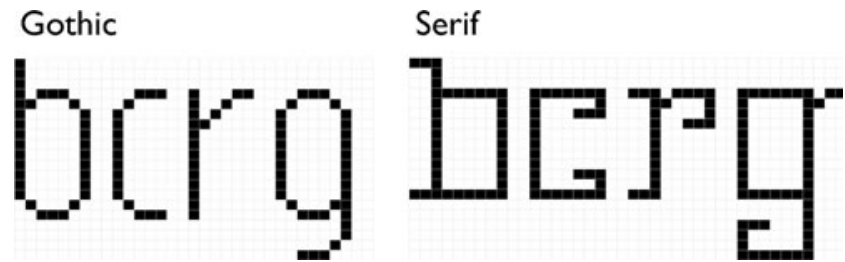

Fig. 4 Examples of the two fonts from Sanocki (1988) 
measured accuracy of identifying letters in strings of three letters each. This experiment involved sans serif letters displayed at a fairly high resolution but varying in a spatial size relation - the size of the ascenders and descenders relative to $\mathrm{x}$-height (termed aspect ratio by Gauthier et al., 2006). Gauthier et al. found that when two fonts were mixed within strings, accuracy of identification was lower than when presented in pure strings. This result replicates negative effects of similar spatial properties found by Sanocki (1991b) with dot matrix letters (see below).

Gauthier et al.'s (2006) first two experiments were also conducted with high resolution letters. Gauthier et al. had observers search for a target letter through large matrices of well-spaced individual letters, with a search task that required serial, letter-by-letter processing. They found that search was faster and more accurate through entirely same-font matrices than when font varied from row to row. Search was even slower and less accurate when font varied within rows. The effects were found with English readers and (typical) Roman fonts (Experiment 1) and with Chinese readers and fonts of Chinese characters (Experiment 2). However, the search task requires a decision about each individual letter, and the overall reaction times may be influenced by decision-level effects that would accumulate across letters.

Walker (2008) used Bookman Bold and Palatino Italic and presented words and nonwords as stimuli. Font varied only between strings. Walker presented the strings in pairs (one above the other), and the observer's task was to indicate whether there was a word present in the pair. Critically, responses were faster when the font was the same for the pair of strings, as compared with different-font pairs. Walker developed the idea that font tuning is the setting of parameters and explored the time course of tuning over trials (see also Sanocki, 1992). Walker's results clearly implicate the processing of font-specific information, and Walker's discussion of how font parameters may change over time is illuminating. However, the pattern of error rates could imply a speed-accuracy trade-off; error rates were somewhat (but not significantly) higher in same-font conditions. This could reflect the trading of accuracy for speed in same-font conditions; consequently, it is not clear that letter activation processes were more efficient here. The pattern of errors is consistent (but again, not significantly so) with decision-level influences in which "samefontness" (perhaps uniformity of style across the pair of strings) is taken as evidence of wordness. This may be analogous to the decision-level effects found in letternonletter decisions (Sanocki, 1992) and well-studied in word-nonword decisions (see, e.g., Neely, 1991).

In summary, there is evidence of same-font over mixedfont advantages with letter strings, both with somewhat coarse dot matrix fonts (Sanocki, 1987, 1988, 1991a, 1991b, 1991c) and with higher resolution fonts (Gauthier et al., 2006), and evidence that font information is retained and used over time (Walker, 2008). There are a number of outstanding questions, however. Two are of particular importance.

What specific properties of fonts are critical for same-font advantages? Is a constellation of differing font properties necessary for regularity effects (see, e.g., Fig. 4), or can specific properties be identified? Existing results have most clearly implicated spatial size parameters. In their Experiment 3, Gauthier et al. (2006) manipulated three different font properties and obtained same-font advantages for only one, the manipulation of aspect ratio mentioned (size of ascenders or descenders, relative to $\mathrm{x}$-height). Two other manipulations had no effect in that experiment: a manipulation of letter slant (letters were tilted left or right with other properties constant) and fill (letters were outlines or filled). This suggests that spatial size parameters may be more important than these other possible font parameters. However, the range of possible font parameters has only been partially explored; remaining parameters include, but are not limited to, extents of terminations, shape of components, and line thickness. Also, spatial size parameters should be further defined and distinguished.

Further analysis of Gauthier et al.'s (2006) positive results for aspect ratio is limited because they did not report results for the separate size ratio fonts. Sanocki (1991b) used a similar manipulation of size ratio. These experiments may provide the strongest evidence for the importance of space size relations. In addition, they provide evidence of a limit in the importance of feature distinctiveness. Sanocki (1991b) first measured the ratio of ascender/descender to xheight in a sample of text fonts and found the average ratio (.38 and .42 for ascenders and descenders to $\mathrm{x}$-height, respectively; $S D \mathrm{~s}=.10)$. Sanocki $(1991 \mathrm{~b})$ then measured identification efficiency for four fonts of briefly presented letter strings that varied in size and size relations. Two were normal fonts with (the same) normal size ratios between parts; one was a small font, and one a large font (twice as large; see Fig. 5). Then two abnormal fonts were created by taking apart letters and mixing the ascenders and descenders of one font size with the bodies of the other size. This resulted in a small/large font (small $\mathrm{x}$-height, large ascenders and descenders; see Fig. 5, second row) and a large/small font (large $\mathrm{x}$-height, small ascenders and descenders). Because ascenders and descenders are generally highly distinctive (e.g., Fiset et al., 2008), the relative amount of distinctive information was increased for the small/large font but reduced for the large/small font. Sanocki (1991b) found that letter identification efficiency was reduced for the less distinctive large/small font, relative to the other three fonts, which were all similar in level of identification. The disadvantage for the large/small font 

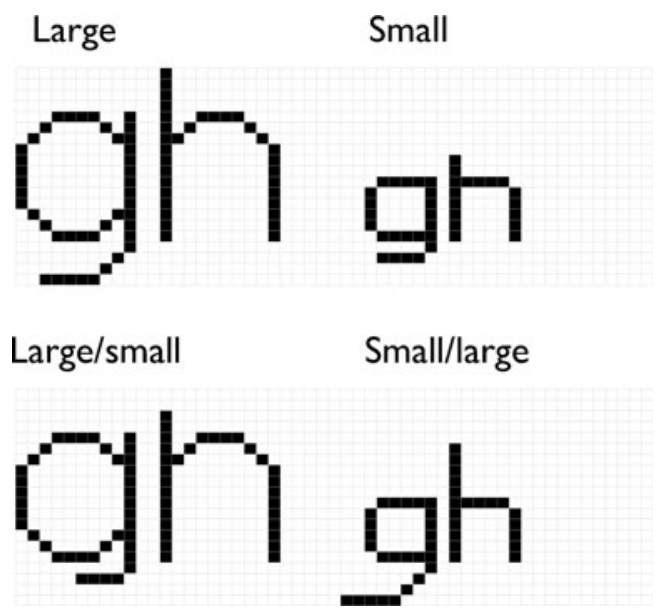

Fig. 5 Normal fonts (top row) and abnormal fonts (bottom) from Sanocki (1991b)

occurred even when pure-font strings were presented throughout blocks. The time course data (Experiment 2) indicated that the reduced efficiency for the large/small letters produced a fairly large decrement in the asymptote of the time course functions. This is consistent with the idea that letter codes were not activated as quickly or as strongly as for the other fonts. The large/small letters may have been more difficult to perceive because their spatial relations were not similar to those of typical fonts and did not strongly activate detectors sensitive to these relations-resulting in less letter-level activation. This raises another important set of issues related to what can be called font typicality.

An additional result was that letter identification efficiency was not improved by an increase in the relative amount of distinctive information, in the small/large font. Even though the ascenders and descenders in this font were relatively large (see Fig. 5), and even though this font was presented as a consistent font throughout blocks of trials (to allow tuning), performance levels were similar to those for the normal fonts. This means that increasing the distinctiveness of the letters within the font did not increase letterprocessing efficiency.

What is the relation between font-mixing effects and font typicality? We begin this section with two perspectives on font mixing and font typicality. The first perspective is the possibility that font mixing has negligible effects on letter identification efficiency under important conditions-when highly legible text fonts of the same apparent size are mixed. We note that good text fonts are, in fact, quite similar to each other when critical size parameters are matched; the fonts may approximate a prototype. However, a second perspective is that the mixing of good fonts clearly has effects when the fonts vary in size relations or spatial relations. The implication is that, at the very least, the study of font tuning is a study of how spatial size relations are processed.
The first perspective arises because, despite the collection of font-mixing studies reviewed, we know of no compelling findings with typical text fonts that are similar in apparent size. Walker (2008) used high-quality fonts, but one was a bold font and the other a fairly readable italic font. Furthermore, there are some concerns about decisionlevel effects in those studies. Gauthier et al. (2006) found their most compelling effects by mixing spatial size relations and producing atypical fonts. The font parameters used by Sanocki $(1987,1988,1991 \mathrm{c})$ extend beyond the range of typical fonts in several ways. Thus, the extent of the mixing effect with typical, high-quality text fonts is an open issue.

Given modern computer technology and the proliferation of fonts, it should be relatively easy to test for font-mixing effects with typical fonts. However, typical text fonts are similar to each other in many ways. It is possible that, when equated for apparent size, there may not be enough variation between them to produce mixed-font disadvantages. For example, consider the text fonts shown in Fig. 3, chosen for their strong stylistic differences. Now let us consider their similarities (Fig. 6a). Comparison of letters from font to font (within the columns of Fig. 6a) reveals strong similarities. In fact, when the letters are superimposed upon each other (Fig. 6b), one can see that their spatial parameters are quite similar. This is true for many popular text fonts. If spatial properties are critical, there may not be enough difference between typical fonts to produce mixing effects when apparent size is equated.

In any case, further exploration of font-mixing effects with a range of fonts is in order. In this exploration process, measures in addition to letter identification efficiency should also be considered, as we suggest below. The similarity of good text fonts to each other, and to a prototypical form, has interesting implications, which are also explored below. a)

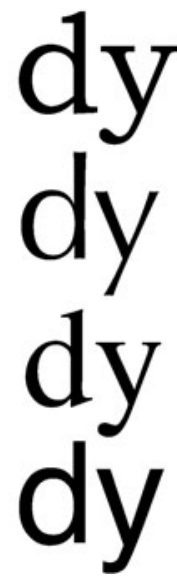

b)

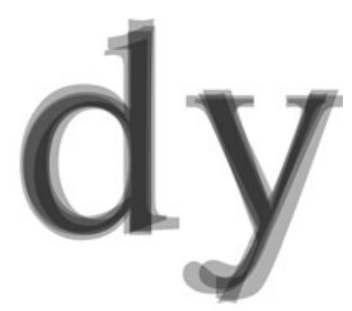

Fig. 6 Similarity of letter shapes: Examples from the four fonts shown in Fig. 3 (a) and their overlap (b) 
Although the existence of mixing effects for similarly sized text fonts is an open question, fonts are size-specific entities in the type world. They have a nominal point size, and there are, of course, real differences in size. The evidence is now clear than when different-size normal fonts are mixed, there are negative effects on letter and word processing. These effects include the negative effects of mixing spatial size relations within letters, studied by Gauthier et al. (2006) and Sanocki (1991b). As noted, the time course functions of Sanocki (1991b) indicate that these negative effects occur on the efficiency of letter activation. And there is another negative effect involving size-the size of letters with normal spatial relations - which we now turn to.

Size variation between letters has produced robust negative effects; however, these may not result from changes in letter identification efficiency. We now discuss those results briefly. Rudnicky and Kolers (1984) mixed large and small letters in reading and found a number of negative effects on reading speed. With normal fonts differing in size, Sanocki (1991b) found that letter identification accuracy was reduced when large and small normal letters were mixed, and only for the smaller letters. A conservative explanation for these negative effects is differences in attentional priority, as will be explained. Finally, recent research suggests that the negative effects of mixing letter case (eXaMpLe; McClelland, 1976) may arise because the differences in the size of upper- and lowercase letters disrupt the grouping of letters into word units; uppercase letters look and function as larger letters and may combine to form competing sublexical groups (Humphreys et al., 2003; Mayall et al., 1997). The attentional priority explanation of these results is that, when large and small letters are mixed within strings, the larger letters are a higher priority for attentional processes that read out abstract letter identity as visual processing is completed (Sanocki, 1991b). The attentional priority explanation was developed in the literature on hierarchical letters - that is, larger letters composed of smaller letters (e.g., Miller, 1981; Navon, 1977; Ward, 1982) - to explain advantages for the larger (global) level in decision. It can be applied to perceiving letters in strings (Sanocki, 1991b) and words. With mixed-case words, differences in size-based priority would disrupt the formation of word units but would encourage (inappropriate) grouping of same-size or samecase letters (see Humphreys et al., 2003). With mixed-sized strings, the presence of large letters makes adjacent smaller letters a lower priority for encoding, especially at string positions that get less attention (rightward positions for English readers; Sanocki, 1991b, Experiment 3). Future research on size mixing should consider effects on letterprocessing efficiency and the further possibility of effects on attention to large and small letters (Sanocki, 1991b).

\section{The microgenesis of letter and word processing}

Evidence of the importance of common letter information can also be found in the time dimension of identification. In most of the experiments discussed, the time course of visual letter processing has been treated as a unitary event. However, it is possible to manipulate phases within the visual-processing time of a letter - to study the microgenesis of letter processing. Such studies reveal an important further property of commonalities, which is that their importance can change over time.

Most theories of the microgenesis of visual perception assume that the earlier phases involve coarse, larger, or more global features and that the representation becomes refined (quickly) over time, with finer details being resolved late in processing (e.g., Broadbent, 1977; Fiset et al., 2009; Navon, 1977; Palmer, 1975; Sanocki, 1991a, 1993, 2001). As was suggested in the introduction, the early phases establish some type of reference frame, such as the spatial envelope of letters. Also, in early phases, larger scale features, such as large letter parts, are likely to be perceptually important. These larger features can function as a commonality that aids letter perception (Sanocki, 1991a). For example, the vertical stroke that is common to $f$ and $t$ is a major structure within the letter. If established early in processing, this structure could be useful in integrating smaller parts, such as the top or bottom curves that distinguish the letters. In a simple study of the microgenesis of letter processing, Sanocki (1991a) presented large common features early in processing as a prime (for 33-67 ms), followed by the entire letter for a similar amount of time and a mask. Observers choose between the pair of similar letters. The primes always involved features common to the forced choice alternatives, providing no distinctive information. Yet they facilitated target identification, relative to no prime and various baseline primes. The results were extended to objects with common features primes, and temporally opposite results were obtained with small distinctive features (benefits for presentation in late processing, but not early processing, in contrast to large common features that had benefits early but not late; Sanocki, 1993, 2001). These results indicate that benefits of common information change over time.

The microgenesis of processing could also be extended to processing letters in words. As we have suggested, an important early phase of processing is the establishment of reference frames for letters; these frames could establish ascender and descender regions, among other properties. Manipulations that disrupt the regular letter frames of a good, normal font are likely to disrupt letter and word identification (e.g., Humphreys et al., 2003; Sanocki, 1991b). This time course perspective may be helpful in understanding when a mixed font will and will not impact 
letter and word identification. For example, consider the mixture of similar-sized good fonts in Fig. 7a. These fonts presumably have similar reference frames (see also Fig. 6b), and the coarse information processed early in time may be very similar across the two fonts. Given that readers can integrate letter information very efficiently within words, a skilled reader needs only some information about each letter to identify the word. This information could come from early processing. That is, coarse information in early processing often may be enough for quick word identification. If so, then only when the fonts are different enough in early coarse spatial information would word identification be slowed down by font mixing. In summary, further studies of the microgenesis of letter and word processing should be illuminating and may further establish the importance of common information in letter processing and reading.

\section{Font typicality, type design, and language}

The similarity of popular text fonts suggests that letter designers have developed, over centuries of research into design, prototype structures of individual letters. In the design field, Johnston described these as essential or structural forms: "the simplest forms that preserve the characteristic structure, distinctiveness, and proportions of each individual letter" (Johnston, 1945, p. 239). Similarity to prototype may be critical for acceptance or popularity of the font, and it may be critical for optimal legibility. Designers may have discovered, through their intuitive research methods, that fonts become less legible if they vary too much from the prototypical structure.

The basis of the prototypical structure of popular text fonts is an open and interesting question. Is the prototypical structure due to the nature of the human visual system? Have designers, by striving for legibility, developed a system that approaches optimality for the human visual

\section{a) The quick brown fox ran through the garden before we could act.}

\section{b) The quick brown fox ran through the garden before we could act.}

\section{c) The quick brown fox ran through the garden before we could act.}

Fig. 7 A sentence in mixed font (a), Bookman Old Style (b), and Optima (c) system? These questions are complex and involve a number of separate issues, but headway is being made.

The parallels between the visual system and the structure of the world have been discussed in depth by theorists for some time (e.g., Lockhead \& Pomerantz 1991; Shepard, 2001). In alphabet design, Changizi, Zhang, Ye, and Shimojo (2006) argued that letters have shapedistinctions analogous to objects in the natural environment, allowing readers to exploit general recognition mechanisms that evolved to efficiently perceive objects. Changizi et al. supported their thesis by finding commonalities in contour configurations across writing systems, nonlinguistic symbols, and natural scenes. In a further study of letter components, Changizi and Shimojo (2005) concluded that writing systems have evolved to balance distinctiveness and uniformity. Parallels between object perception and letter design were also studied by Lanthier, Risko, Stolz and Besner (2009). In object perception, vertices are argued to be more important than midsegment contours, because of their utility in distinguishing object shapes (e.g., Biederman, 1987); Lanthier et al. showed that vertices are also more important than midsegments for letter identification.

The research just discussed emphasizes distinctiveness. However, the richer framework we argue for includes the translation from deeper or more essential levels to the details of letters and emphasizes that uniformity of this translation is also important (see also Walker, 2008). Similar translations may be involved in speech perception, where deeper phonetic relations are modified by the talker (e.g., Martin, Mullennix, Pisoni, \& Summers, 1989). For example, within a single talker, the acoustic pattern of one vowel is distinct from other vowels, but one vowel of a talker overlaps with the acoustic pattern of a different vowel spoken by another talker. We are able to identify vowels correctly across different talkers because we process the systematic covariation in signal properties (talker characteristics) to separate phonetic categories (Nearey, 1989). The talker-dependent variation applies to all vowels almost uniformly. Talker regularity effects are also found in that mixing talkers within a block of trials leads to vowel identification that is worse than that for a single talker (Nearey, 1989).

Given the importance of deep versus surface structure in letter perception and speech perception, could it also apply to object perception? In modern times, the details of manufactured objects often depend on the intended style. Would a chair of one style (e.g., early American) be perceived less efficiently in the context of another style (e.g., an otherwise modern Scandinavian room)? This is an open question; if such influences exist, models of object recognition that emphasize only distinctiveness would have to be modified. 


\section{Measuring the efficiency of the front end of reading: Judgments by the visually educated}

We have argued that measuring the identification of letters in strings is a good way of assessing the efficiency of reading's front end. However, there is a more sensitive and yet simpler measure that could be further explored-the "ease" or "enjoyment" of reading, assessed subjectively by a visually educated reader. This is a primary measure that type designers and typographers develop and refine as they build up design skill.

The reader is invited to consider the ease of reading the sentence in Fig. 7 a, written in alternating letters from two fonts (i.e., a mixed font). While it is easy to ignore the modest "noise" and read the sentence, is the visual experience as pleasant as with pure fonts (Fig. 7b, c)? Would it be pleasant to read an entire book in mixed font? Measurements of subjective ease could be quite sensitive.

There may be more at stake here than ease of reading, however. Researchers have known for some time that readers allocate processing resources at multiple levels during reading and can alter their allocation strategy (e.g., Laberge \& Samuels, 1974). Mixed font may be like visual noise. When visual noise is present, readers can still read quickly, but at a cost of resources and comprehension at higher levels (e.g., Gao, Stine-Morrow, Noh, \& Eskew, 2011). Measures of ease and enjoyment may be sensitive to visual noise and be more sensitive to font-mixing effects than are measures of letter identification efficiency. Such measures could be scientifically useful, in combination with objective measures.

Combined objective and subjective measurement could also be used to test a critical assumption motivating type design, that subjective measurements are informative about visual processing. We have argued that type designer's judgments appear to be valid in general, but this is an assumption that should be examined.

\section{Conclusions}

Our main goal in this article has been to stimulate further thinking about the front end of reading - the process of letter identification. Although distinctive features are psychologically important aspects of letter identification, we argue that their details are not arbitrary. A richer approach to letter identification involves distinctiveness together with the commonalities of letters - uniformities from letter to letter within a font, pertaining to spatial and size relations - and perhaps other stylistic details that characterize a font. Commonalities may be important for establishing spatial reference frames for letters and may help to define a family of objects for efficient identification. Font-mixing research begins to provide evidence that commonalities influence letter identification efficiency. However, critical questions remain to be answered. A variety of research approaches should be considered, including studies of the microgenesis of processing and, possibly, subjective measures such as those used by type designers.

The openness of these issues means that vision researchers should not be quick to propose changes in alphabet design to the larger world. Alphabet design is a complex topic, and it can be a fascinating topic for vision science, one that we can learn from. However, the topic should be reasonably understood before we can safely influence visual culture.

Acknowledgments The authors thank Gregg Oden for helping to inspire the initial research and for helpful comments on this manuscript, and Rosanna Traina for assisting with illustrations. MCD was supported by the University of Reading's Research Endowment Trust Fund.

\section{References}

Adams, D. (1989). abcdefg, a better constraint driven environment for font generation. In J. André \& R. Hersch (Eds.), Raster imaging and digital typography (pp. 54-70). Cambridge: Cambridge University Press.

Arditi, A., \& Cho, J. (2007). Letter case and text legibility in normal and low vision. Vision Research, 47, 2499-2505.

Baines, P., \& Haslam, A. (2005). Type \& typography (2nd ed.). London: Laurence King.

Beier, S., \& Larson, K. (2010). Design improvements for frequently misrecognized letters. Information Design Journal, 18, 118-137.

Besner, D., Davelaar, E., Alcott, D., \& Parry, P. (1984). Wholistic reading of alphabetic print: Evidence from the FDM and the FBI. In L. Henderson (Ed.), Orthographies and reading: Perspectives from cognitive psychology, neuropsychology and linguistics (pp. 121-135). Hillsdale, NJ: Erlbaum.

Biederman, I. (1987). Recognition-by-components: A theory of human image under-standing. Psychological Review, 94, 115-147.

Bouma, H. (1971). Visual recognition of isolated lower case letters. Vision Research, 11, 459-474. doi:10.1016/0042-6989(71)90087-3

Broadbent, D. E. (1977). Levels, hierarchies, and the locus of control. Quarterly Journal of Experimental Psychology, 29, 181-201.

Carter, R., Day, B., \& Meggs, P. (1985). Typographic design: Form and communication. New York: Van Nostrand Reinhold.

Changizi, M. A., \& Shimojo, S. (2005). Character complexity and redundancy in writing systems over human history. Proceedings of the Royal Society B, 272, 267-275.

Changizi, M. A., Zhang, Q., Ye, H., \& Shimojo, S. (2006). The structures of letters and symbols throughout human history are selected to match those found in objects in natural scenes. The American Naturalist, 167, E117-E139.

Cheng, K. (2005). Designing type. New Haven, CT: Yale University Press.

Chung, S. T. L. (2004). Reading speed benefits from increased vertical word spacing in normal peripheral vision. Optometry and Vision Science, 81, 525-535.

Corcoran, D. W. J., \& Rouse, R. O. (1970). An aspect of perceptual organization involved in reading typed and handwritten words. Quarterly Journal of Experimental Psychology, 22, 526-530.

Davis, C. J., \& Bowers, J. S. (2006). Contrasting five different theories of letter position coding: Evidence from orthographic similarity effects. 
Journal of Experimental Psychology: Human Perception and Performance, 32, 535-557. doi:10.1037/0096-1523.32.3.535

Downing, J. (1967). What's wrong with the i.t.a.? Phi Delta Kappa, 262-266.

Finkbeiner, M., \& Coltheart, M. (2009). Letter recognition: From perception to representation. Cognitive Neuropsychology, 26, 1-6. doi:10.1080/02643290902905294

Fiset, D., Blais, C., Arguin, M., Tadros, K., Éthier-Majcher, C., Bub, D., et al. (2009). The spatio-temporal dynamics of visual letter recognition. Cognitive Neuropsychology, 26, 23-35. doi:10.1080/ 02643290802421160

Fiset, D., Blais, C., Éthier-Majcher, C., Arguin, M., Bub, D., \& Gosselin, F. (2008). Features for Identification of uppercase and lowercase letters. Psychological Science, 19, 1161-1168. doi:10.1111/j.1467-9280.2008.02218.x

Friedman, R. (1980). Identity without form: Abstract representations of letters. Perception \& Psychophysics, 28, 53-60.

Gao, X., Stine-Morrow, E. A. L., Noh, S. R., \& Eskew, R. T., Jr. (2011). Visual noise disrupts conceptual integration in reading. Psychonomic Bulletin \& Review, 18, 83-88.

Gauthier, I., Wong, A. C.-N., Hayward, W. G., \& Cheung, O. S. (2006). Font tuning associated with expertise in letter perception. Perception, 35, 541-559. doi:10.1068/p5313

Gervais, M. J., Harvey, L. O., \& Roberts, J. O. (1984). Identification confusions among letters of the alphabet. Journal of Experimental Psychology: Human Perception and Performance, 10, 655666.

Gibson, E. J. (1969). Principles of perceptual learning and development. Englewood Cliffs, NJ: Prentice-Hall.

Gilmore, G. C. (1985). Letters are visual-stimuli: A comment on the use of confusion matrices. Perception \& Psychophysics, 37, 389-390.

Goldinger, S. D., Azuma, T., Kleider, H. M., \& Holmes, V. M. (2003). Font-specific memory: More than meets the eye? In J. S. Bowers \& C. J. Marsolek (Eds.), Rethinking implicit memory (pp. 157196). Oxford: Oxford University Press.

Gomez, P., Ratcliff, R., \& Perea, M. (2008). The overlap model: A model of letter position coding. Psychological Review, 115, 577600. doi:10.1037/a0012667

Gosselin, F. \& Tjan, B. S. (2008, May). The past, present, and future of the written word. [Symposium Summary]. Symposium presented at the 8th Annual Meeting of the Vision Sciences Society, Naples, FL.

Grainger, J. (2008). Cracking the orthographic code: An introduction. Language and Cognitive Processes, 23, 1-35. doi:10.1080/ 01690960701578013

Grainger, J., Rey, A., \& Dufau, S. (2008). Letter perception: From pixels to pandemonium. Trends in Cognitive Sciences, 12, 381387. doi:10.1016/j.tics.2008.06.006

Harris, J. (1973). Confusions in letter recognition. Printing Technology, 17(2), 29-34

Humphreys, G. W., Mayall, K. A., \& Cooper, A. C. G. (2003). The PIG in sPrInG: Evidence onletter grouping from the reading of buried words. Psychonomic Bulletin \& Review, 10, 939-946.

Johnston, E. (1945). Writing \& illuminating, \& lettering (21st reprint). London: Pitman.

Juhasz, B. J., Pollatsek, A., Hyönä, J., Drieghe, D., \& Rayner, K. (2009). Parafoveal processing within and between words. Quarterly Journal of Experimental Psychology, 62, 1356-1376. doi:10.1080/17470210802400010

Keren, G., \& Baggen, S. (1981). Recognition models of alphanumeric characters. Perception \& Psychophysics, 29, 234-246.

Knuth, D. E. (1982). The concept of a meta-font. Visible Language, 16, 3-27.

Kolers, P. A. (1969). Clues to a letter's recognition: Implications for the design of characters. Journal of Typographical Research, 3, $145-168$.
LaBerge, D., \& Samuels, S. J. (1974). Toward a theory of automatic information processing in reading. Cognitive Psychology, 6, 293323.

Lanthier, S. N., Risko, E. F., Stolz, J. A., \& Besner, D. (2009). Not all visual features are created equal: Early processing in letter and word recognition. Psychonomic Bulletin \& Review, 16, 67-73. doi:10.3758/PBR.16.1.67

Livne, T., \& Sagi, D. (2007). Configuration influence on crowding. Journal of Vision, 7(2, Art. 4), 1-12, Retrieved from http:// journalofvision.org/7/2/4/. doi:10.1167/7.2.4

Lockhead, G. R., \& Crist, W. B. (1980). Making letters distinctive. Journal of Educational Psychology, 72, 483-493.

Lockhead, G. R., \& Pomerantz, J. R. (1991). The perception of structure. Washington, DC: American Psychological Association.

Luna, P. (1992). Understanding type for desktop publishing. London: Blueprint.

Marr, D. (1982). Vision. San Fransisco: Freeman.

Martin, C. S., Mullennix, J. W., Pisoni, D. B., \& Summers, W. V. (1989). Effects of talker variability on recall of spoken word lists. Journal of Experimental Psychology: Learning, Memory, and Cognition, 15, 676-684.

Massaro, D. W., \& Sanocki, T. (1993). Visual information processing in reading. In D. Willows, R. Kruck, \& E. Corcos (Eds.), Visual processes in reading and reading disabilities (pp. 139-161). Hillsdale, NJ: Erlbaum.

Massaro, D. W., \& Schmuller, J. (1975). Visual features, preperceptual storage and processing time in reading. In D. W. Masaro (Ed.), Understanding language (pp. 207-239). New York: Academic Press.

Mayall, K., Humphreys, G. W., \& Olson, A. (1997). Disruption to word or letter processing? The origins of case-mixing effects. Journal of Experimental Psychology: Learning, Memory, and Cognition, 23, 1275-1286.

McClelland, J. L. (1976). Preliminary letter identification in the perception of words and nonwords. Journal of Experimental Psychology: Human Perception and Performance, 2, 80-91.

McClelland, J. L., \& Rumelhart, D. E. (1981). An interactive model of context effects in letter perception: Part 1. An account of basic findings. Psychological Review, 80, 375-407.

Miller, J. (1981). Global precedence in attention and decision. Journal of Experimental Psychology: Human Perception and Performance, 9, 1161-1174.

Mozer, M. C. (1983). Letter migration in word perception. Journal of Experimental Psychology: Human Perception and Performance, 9, 531-546. doi:10.1037/0096-1523.9.4.531

Navon, D. (1977). Forest before trees: The precedence of global features in visual perception. CognitivePsychology, 9, 353-383.

Nearey, T. M. (1989). Static, dynamic, and relational properties in vowel perception. Journal of the Acoustical Society of America, $85,2088-2113$.

Neely, J. H. (1991). Semantic priming effects in visual word recognition: A selective review of current findings and theories. In D. Besner \& G. Humphreys (Eds.), Basic processes in reading: Visual word recognition (pp. 264-336). Hillsdale, NJ: Erlbaum.

Oden, G. C. (1979). A fuzzy logical model of letter identification. Journal of Experimental Psychology: Human Perception and Performance, 5, 336-352.

Oden, G. C. (1984). Dependence, independence, and emergence of word features. Journal of Experimental Psychology: Human Perception and Performance, 10, 394-405.

Oden, G. C., Rueckl, J. G., \& Sanocki, T. (1991). Making sentences make sense, or words to that effect. In G. B. Simpson (Ed.), Understanding word and sentence (pp. 285-303). Amsterdam: North-Holland.

Palmer, S. E. (1975). Visual perception and knowledge: Notes on a model of sensory-cognitive interaction. In D. A. Norman \& D. E. 
Rumelhart (Eds.), Explorations in Cognition (pp. 279-307). San Francisco: Freeman.

Paterson, D. G., \& Tinker, M. A. (1940). Influence of line width on eye movements. Journal of Experimental Psychology, 27, 572-577.

Pelli, D. G., Burns, C. W., Farell, B., \& Moore-Page, D. C. (2006). Feature detection and letter identification. Vision Research, 46, 4646-4674. doi:10.1016/j.visres.2006.04.023

Pelli, D. G., \& Farell, B. (1999). Why use noise? Journal of the Optical Society of America A, 16, 647-653.

Pelli, D. G., Farell, B., \& Moore, D. C. (2003). The remarkable inefficiency of word recognition. Nature, 423, 752-756. doi:10.1038/nature01516

Pelli, D. G., Palomares, M., \& Majaj, N. J. (2004). Crowding is unlike ordinary masking: Distinguishing feature integration from detection. Journal of Vision, 4(12), 1136-1169.

Pelli, D. G., Tillman, K. A., Freeman, J., Su, M., Berger, T. D., \& Majaj, N. J. (2007). Crowding and eccentricity determine reading rate. Journal of Vision, 7(2, Art. 20, 1-36. http://journalofvision.org/7/2/20/

Pinker, S. (1984). Visual cognition: An introduction. Cognition, 18, 1-63.

Pittman, J., \& St. John, J. R. (1969). Alphabets and reading: The Initial Teaching Alphabet. New York: Pittman.

Reicher, G. M. (1969). Perceptual recognition as a function of meaningfulness of stimulus material. Journal of Experimental Psychology, 81, 275-280.

Rudnicky, A. I., \& Kolers, P. A. (1984). Size and case of type as stimuli in reading. Journal of Experimental Psychology: Human Perception and Performance, 10, 231-249.

Saarela, T. P., Sayim, B., Westheimer, G., \& Herzog M. H. (2009). Global stimulus configuration modulates crowding. Journal of Vision, 9(2, Art. 5), 1-11, http://www.journalofvision.org/content/ 9/2/5. doi: $10.1167 / 9.2 .5$

Sanocki, T. (1987). Visual knowledge underlying letter perception: Font-specific schematic tuning. Journal of Experimental Psychology: Human Perception and Performance, 13, 267278.

Sanocki, T. (1988). Font regularity constraints on the process of letter recognition. Journal of Experimental Psychology: Human Perception and Performance, 14, 472-480.

Sanocki, T. (1991a). Effects of early common features on form recognition. Perception \& Psychophysics, 50, 490-497.

Sanocki, T. (1991b). Intrapattern and interpattern relations in letter recognition. Journal of Experimental Psychology: Human Perception and Performance, 17, 924-941.

Sanocki, T. (1991c). Looking for a structural network: Effects of changing size and style on letter recognition. Perception, 20, $529-541$.
Sanocki, T. (1992). Effects of font- and letter-specific experience on the perceptual processing of letters. American Journal of Psychology, 105, 435-458.

Sanocki, T. (1993). Time course of object identification: Evidence for a global-to-local contingency. Journal of Experimental Psychology: Human Perception and Performance, 19, 878-898.

Sanocki, T. (1999). Constructing structural descriptions. Visual Cognition, 6, 299-318.

Sanocki, T. (2001). Interaction of scale and time during object recognition. Journal of Experimental Psychology: Human Perception and Performance, 27, 290-302.

Seymour, P. H. K., \& Jack, M. V. (1978). Effects of visual familiarity on same and differ-ent decision-processes. Quarterly Journal of Experimental Psychology, 30, 455-469.

Shepard, R. N. (2001). Perceptual-cognitive universals as reflections of the world. Behavioral and Brain Sciences, 24, 581-601.

Spencer, H. (1968). The visible word. London: Royal College of Art.

Spinelli, D., De Luca, M., Filippo, G. D., Mancini, M., Martelli, M., \& Zoccolotti, P. (2005). Length effect in word naming in reading: Role of reading experience and reading deficit in Italian readers. Developmental Neuropsychology, 27, 217-235.

Sternberg, S. (1969). The discovery of processing stages: Extensions of Donders' method. Acta Psychologica, 30, 276-315.

Stuart, J. A., \& Burian, H. M. (1962). A study of separation difficulty: Its relationship to visual acuity in normal and amblyopic eyes. American Journal of Ophthalmology, 53, 471-477.

Tanner, W. P., Jr., \& Birdsall, T. G. (1958). Definitions of d' and $n$ as psychophysical measures. Journal of the Acoustical Society of America, 30, 922-928.

Tinker, M. A. (1963). Legibility of print. Ames: Iowa State University Press.

Tinker, M. A., \& Paterson, D. G. (1928). Influence of type form on speed of reading. Journal of Applied Psychology, 12, 359-368.

Walker, P. (2008). Font tuning: A review and new experimental evidence. Visual Cognition, 16, 1022-1058. doi:10.1080/ 13506280701535924

Ward, L. M. (1982). Determinants of attention to local and global features of visual forms. Journal of Experimental Psychology: Human Perception and Performance, 8, 562-581.

Wheeler, D. D. (1970). Processes in word recognition. Cognitive Psychology, 1, 59-85.

White, S. J., Johnson, R. L., Liversedge, S. P., \& Rayner, K. (2008). Eye movements when reading transposed text: The importance of word-beginning letters. Journal of Experimental Psychology: Human Perception and Performance, 34, 1261-1276. doi:10.1037/0096-1523.34.5.1261 